\title{
IRST SiPM characterizations and application studies
}

\author{
G.Pauletta ${ }^{1}$, D.Cauz and C. Del Papa \\ University of Udine \\ Dept. Of Physics, Univ. of Udine, I-33100 Italy \\ E-mail: pauletta@ud.infn.it
}

\author{
V.Bonvicini, L.Bosisio, S. Ciano, A.Driutti, A.Penzo, I.Rashevskaya, \\ A. Vacchi, E. Vallazza \\ INFN Trieste, \\ Area di Ricerca, Padriciano 99 I-34127, Italy \\ E-mail: walter.bonvicini@ts.infn.it
}

\section{C.Piemonte}

FBK-irst

Povo di Trento,Trento, I-38050 Italy

E-mail: piemonte@itc.it

\begin{abstract}
This paper reports on work undertaken, in collaboration with ITC-IRST at Trento, to characterize and test the silicon photomultiplers produced by them, with a view to their future application in high energy and astrophysics experiments. Results of static and dynamic measurents with various IRST devices under controlled climatic conditions, together with measurements with SiPMs from other distributors are reported and discussed with emphasis on progress in the understanding of operational principles and the reduction of noise. Results from the test beam application of the SiPMs are also reported and future plans are discussed.
\end{abstract}

International workshop on new photon-detectors PD07

Kobe University, Kobe, Japan

27-29 June, 2007

\footnotetext{
${ }^{1}$ Speaker
} 


\section{Introduction}

Since the idea of segmenting geiger-mode avalanche photodiodes (GMAPDs) in order to create high-gain silicon-based photodetectors, which we refer to as Silicon Photomultipliers (SiPMs), first emerged [1,2], their development has been undertaken world-wide by several enterprises and institutions. In Italy, this was done at ITC-IRST ${ }^{\dagger}$ [3] in Trento with the support of the INFN $N^{\ddagger}$.

Though the potential of these photo-detectors has been clear since their emergence, much work still needs to be done towards a detailed understanding of their characteristics. Such work is necessarily application-dependent as will be their ensuing evolution. SiPM development at IRST was initially motivated to a large degree by medical applications (PET) while the work reported in this paper focuses on applications to particle physics and astrophysics. Of particular interest to us at the moment is the application of these photo-sensors to calorimetry, large area scintillator-based muon counters and scintillation fiber trackers. Their application to space experiments for UHECR detection and the next generation of large-area, ground-based x-ray telescopes will also be considered. Given a long-standing collaboration between IRST and the section of the INFN based at Trieste in the development of silicon detectors our group is well-placed to contribute to the understanding and to the development of these devices.

The IRST devices are characterized by very shallow junctions, antireflective coating (AR) optimized for short wavelengths $(\sim 400 \mathrm{~nm})$ and polysilicon quenching resistors [3,4]. The baseline model is characterized by a $1 \mathrm{~mm}^{2}$ square geometry comprising 25 by 25 micro-cells, each with an area of 40 by $40 \mu^{2}$. Their design was not optimized for photon detection efficiency (PDE) and the geometric efficiency (sensitive area/insensitive area), also known as the "fill factor" was relatively small $(\sim 20 \%)$ (it has been more than doubled in the latest batch produced). The principal characteristics which affect our applications are gain, noise (dark count, after-pulsing and optical cross-talk), photo-detection efficiency (PDE), dynamic range, timing characteristics (rise-time, resolution and recovery time), radiation hardness and sensitivity to magnetic fields. Thorough device characterization must include a study of all these characteristics and our program of activity is being set up to do so. The

\footnotetext{
${ }^{\dagger}$ The institute for technological research and development (IRST) of the Istituto Trentino di Cultura ( now Bruno Kessler Foundation)

${ }^{\ddagger}$ The National Institute for Nuclear Physics ( INFN)
} 
optimization of packaging and readout electronics as a function of application will also be high on our list of priorities.

We first report on static measurements and on dynamic measurements performed in the dark at Trieste and Udine. These measurements are compared to and complemented by measurements performed at IRST. All these measurements were perfomed on devices with baseline geometry. Some of the most recent IRST SiPMs are then presented and preliminary tests of their performance are referred to. Finally, some results of the application of IRST SiPMs to prototype detectors at various test beam sites are briefly described.

\section{Characterizations}

\subsection{Static measurements}

I-V measurements afford a rapid test of functionality, uniformity and stability. They are also sensitive to the principal device properties. I-V measurements performed at Udine are shown in fig. 1, together with the the breakdown voltages extracted from them and

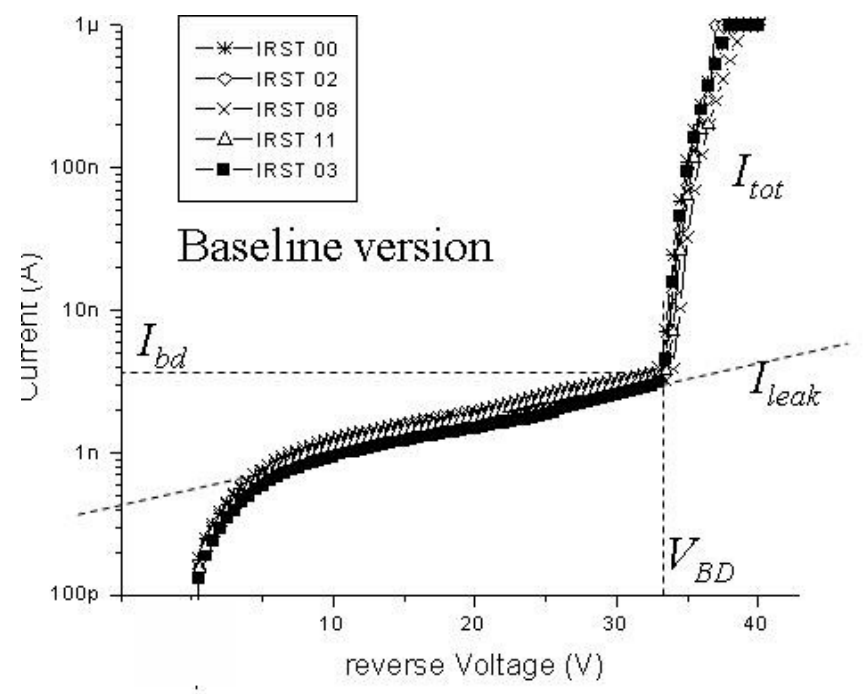

\begin{tabular}{|c|c|c|}
\hline SiPM & Vbd (N) & Ibd (nA) \\
IRST-00 & 32,5 & 3,6 \\
IRST-02 & 33,0 & 3,6 \\
IRST-03 & 33,0 & 3,1 \\
IRST-08 & 33,5 & 3,2 \\
IRST-11 & 33,5 & 3,8
\end{tabular}

Figure 1: I-V measurements for IRST SiPMs

the corresponding reverse currents in the adjoining table. As suggested in [5], the dark current $I_{d c}=I_{\text {tot }}-I_{\text {leak }}$ (assuming a linearly increasing leakage current) above $\mathrm{V}_{\mathrm{BD}}$ is seen to increase as the square of the of the voltage above breakdown $\Delta V=V-V_{B D}$ so that, given one of the parameters DC (dark count) $V_{B D}, R_{Q}$ (quenching resistance) and $G$ (gain), the application of this model to I-V measurements can be used to extract accurate information regarding the other three [5]. Comparison with measurements of devices from other sources [6] served to illustrate the relative uniformity 
of IRST SiPMs. Measurements of the diode capacitance $\mathrm{C}_{\mathrm{D}}$ were also performed with results $(\sim 90 \mathrm{fF})$ which are compatible with expectations based on structural considerations [5]. Forward biased measurements served to determine $R_{Q}: \sim 350 \mathrm{k} \Omega$ for IRST SiPMs compared to considerably larger (2.5 to 50 times) quenching resistance of devices produced with the alternative metal resistive semiconductor (MRS) technology.

\subsection{Dynamic measurements}

All measurements reported here were performed in the dark using a $\sim 20 \mathrm{~dB}, 3.5 \mathrm{GHz}$ amplifier [7 ]. The signal from a MRS device ${ }^{1}\left(\mathrm{R}_{\mathrm{Q}}=0.9 \mathrm{M} \Omega\right.$ ), measured under these conditions is shown in figure 2. Signals from an IRST SiPM (measured with greater amplification) are shown alongside for comparison. The difference in decay time of the IRST signal is compatible with their shorter time constant $\mathrm{R}_{\mathrm{Q}} \mathrm{C}_{\mathrm{D}}(\sim 30 \mathrm{~ns})$.
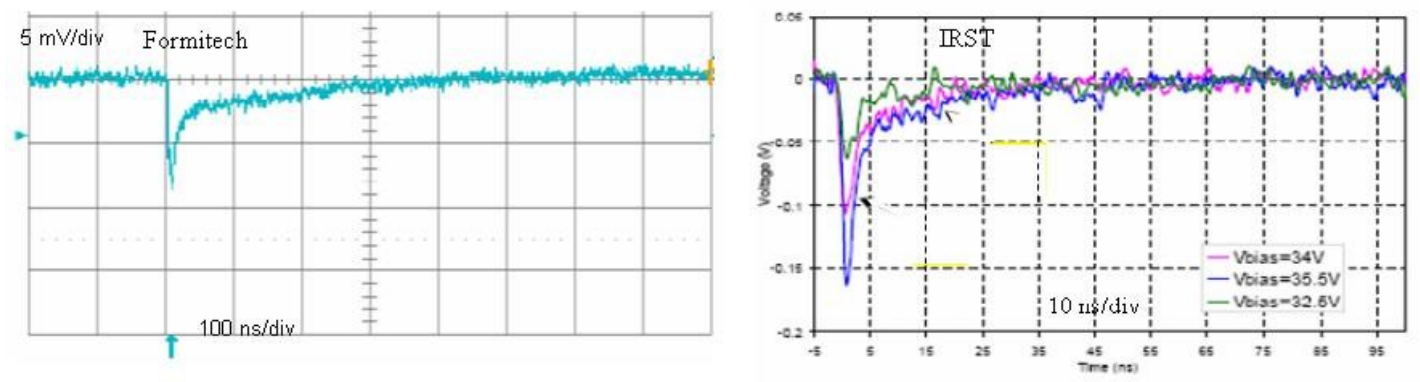

Figure 2: Comparison between pulse shape from an MRS SiPM (Forimtech) and a polysilicon SiPM from IRST

Measurements of dark count and signal amplitude as a function of bias voltage and temperature were performed, both at room temperature and in a climatic chamber with temperature and humidity control. The amplifier was located outside the chamber and the signal was transported to it by means of a special $50 \Omega$ cable with an operating frequency of $18 \mathrm{GHz}$. Measurements of dark count as a function of over-voltage $(\Delta \mathrm{V})$ at room temperature are shown in figure 3 for several IRST SiPMs (A1, B1,B2 D1 and D2). These devices came from different wafers whose process characteristics were varied to reduce the dark current (DC).

\footnotetext{
${ }^{1}$ distributed by Forimtech S.A.
} 


\section{IRST, Dark count (room T)}

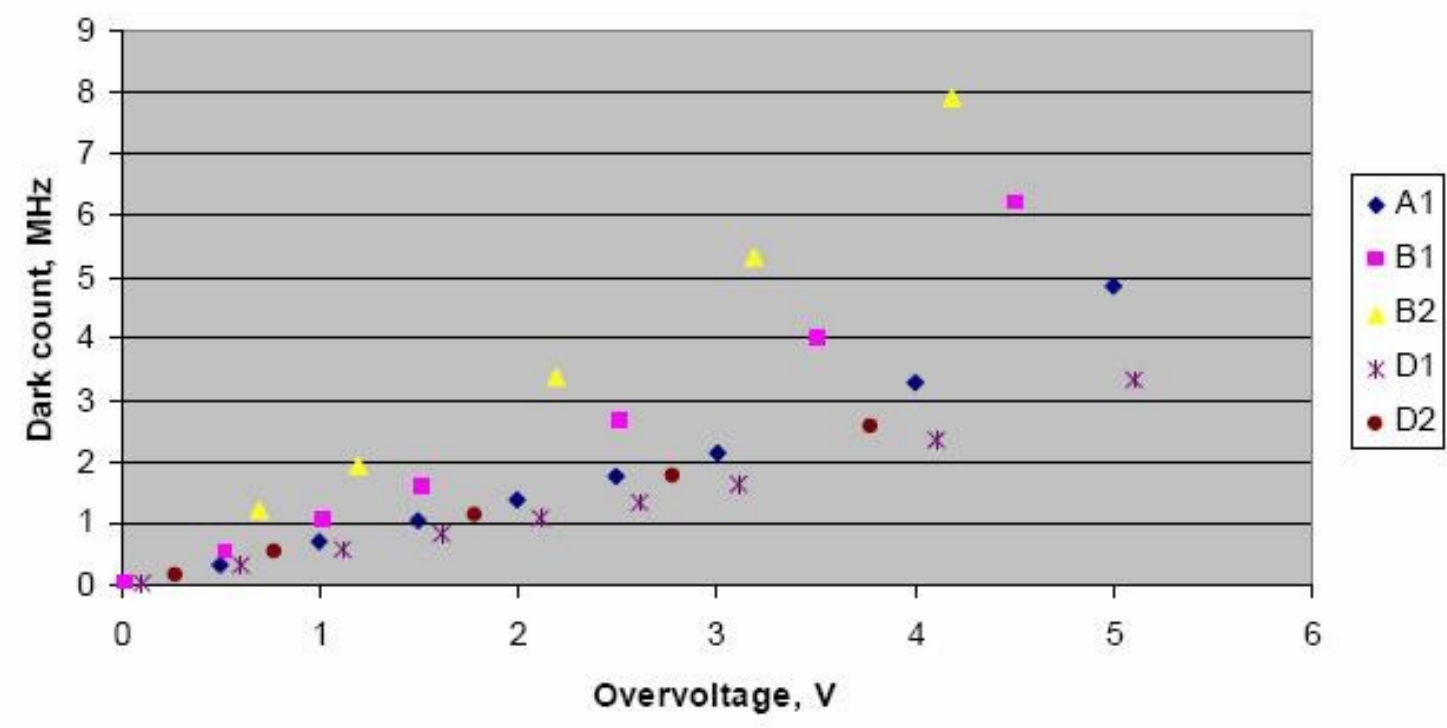

Figure 3: Measurements of DC vs over-voltage for different IRST SiPMs

These curves reflect progress in the reduction of the dark count DC. They were obtained by measuring the $\mathrm{DC}$ as a function of bias voltage $\mathrm{V}$ and then subtracting $\mathrm{V}_{\mathrm{BD}}$ (which can be deduced from their intercept with $\mathrm{V}$ ). Because the gain is proportional to $\mathrm{V}_{\mathrm{BD}}$, the dependence of the breakdown voltage on the temperature is an important parameter. $\mathrm{V}_{\mathrm{BD}}$ was found to be $76 \mathrm{mV}$ per degree centigrade, in accordance with the values quoted by IRST. Measurements of DC as a function of temperature for one of these devices are shown in figure 4 . Signal 


\section{IRST_D1, Dark count}

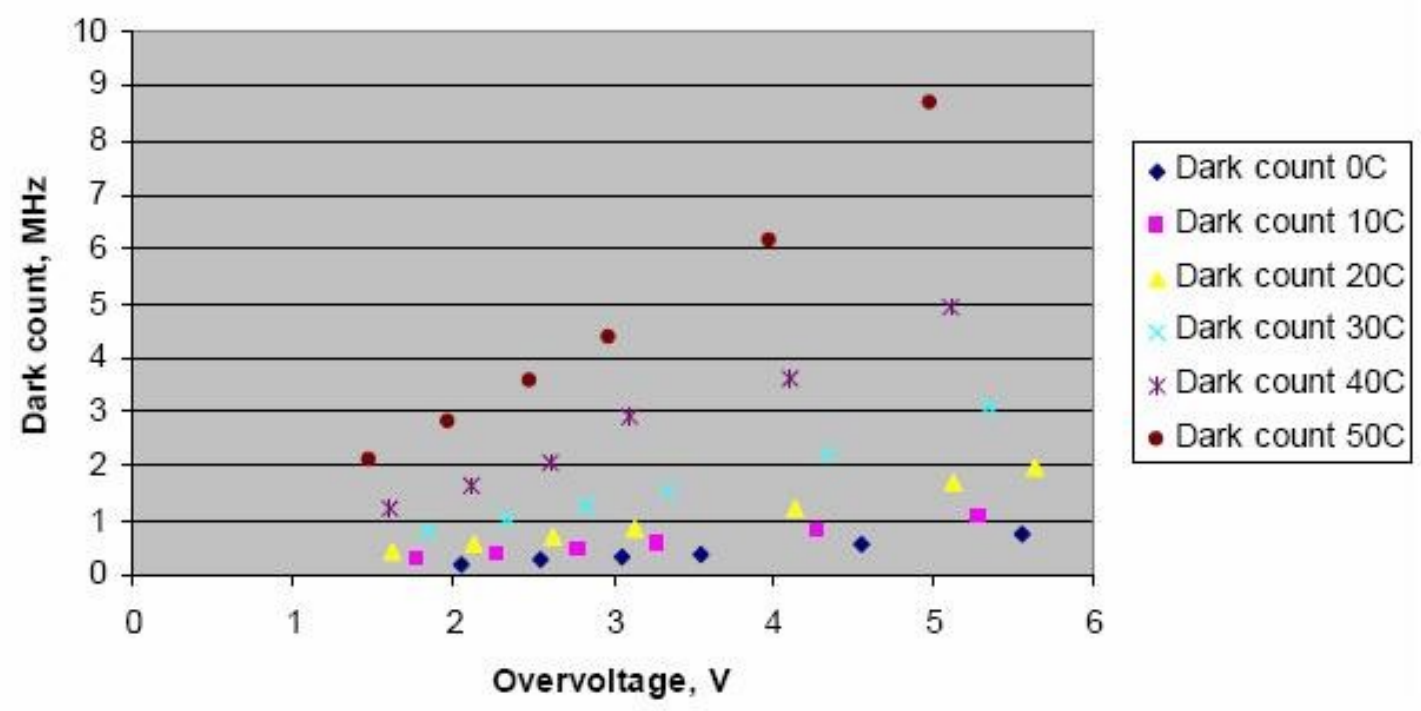
SiPMs

Figure 4: Dark count as a function of overvoltage for one (D1) of the IRST

amplitudes were also measured as a function of bias voltage (their intercept with $\mathrm{V}$ affords another means of determiing $\mathrm{V}_{\mathrm{BD}}$ ) at different temperatures and the temperature-dependences were found to be compatible with a negative temperature dependence of the gain. Details of these measurements were presented recently at Perugia [8] A temperature dependence of the gain of these devices, reported by IRST at Perugia [9], is $G=(-0.4 \widetilde{\sigma}+2) \times 10^{6}$. Some other results reported by Piemonte at that meeting are summarized below in order to update the reader on the current status.

\subsection{After-pulsing and cross-talk}

Charge integration of the signals produces a spectrum with well-known tails on the high side [5]. These are interpreted as consequences of afterpulsing and optical cross-talk. Because cross-talk is initiated by photons emitted in the course of an avalanche in a neighboring cell, associated signals are virtually simultaneous with the primary discharge. By restricting the integration of pulses occuring a short time (e.g. <10 ns) after the beginning of the primary discharge, one retains sensitivity to the cross-talk component of the high-end tail while excluding the after-pulses which are suppressed by quenching. This apparent in figure 5 where a number of primary pulses, which were followed by after-pulses, are 
collected to illustrate how the amplitude of the after-pulses is reduced by quenching near the primary pulse. In this way, it has been estimated [9] that cross-talk contributes no more than $5 \%$ at $4 \mathrm{~V}$ above breakdown.

Figure 5 also serves to show that the probability of afterpulsing is significant for a time interval of less than $\sim 50 \mathrm{~ns}$ after the primary pulse so that, by integrating the charge over a longer interval, one can evaluate the after-pulsing probability which is plotted as a function bias voltage on the right-hand side of figure 5. The parabolic dependence of this probability on the bias has been explained [5] in terms of the linear increases with $\Delta \mathrm{V}$ of both the dark current and the avalanche triggering probability and these conclusions are supported by studies of single microcell structures, where cross-talk does not contribute.
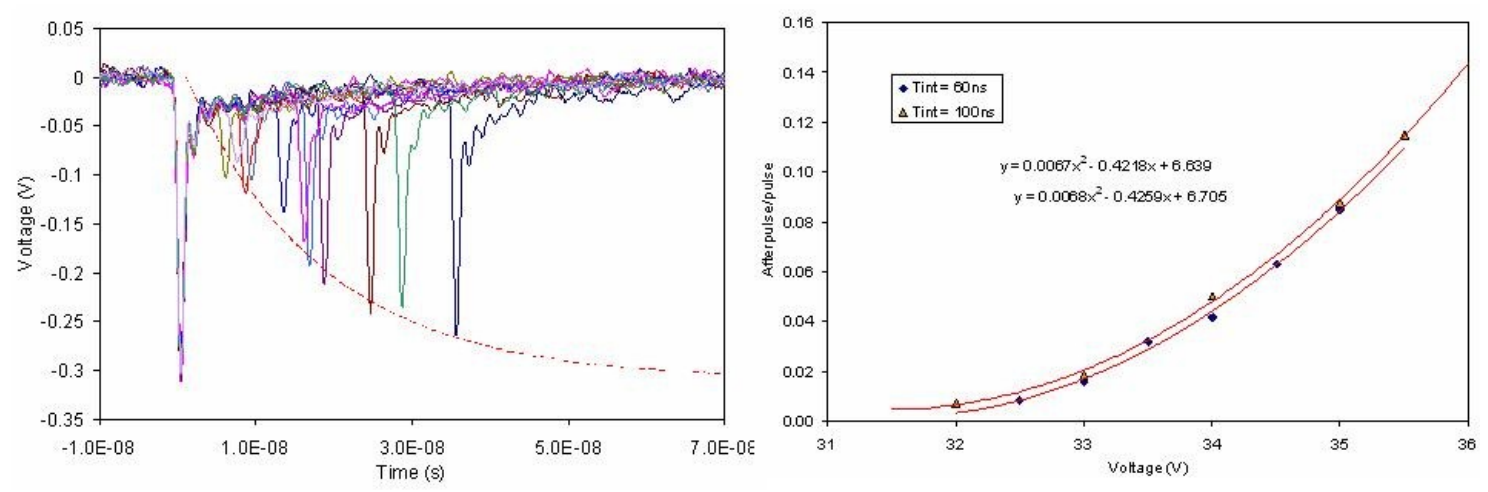

Figure 5: Primary pulse followed by afte-pulses (left) and aferpulsing probability as a fuction of bias voltageV (right).

Measurements of the photo-detection efficiency (for devices with baseline geometry) were also reported by Piemonte at Perugia[8]. PDEs were found to peak at $14 \%$ at wavelengths ranging between 500 and $650 \mathrm{~nm}$ at $4 \mathrm{~V}$ over breakdown voltage. These are relativlely small because the geometrical efficiency of the base-line devices is only $20 \%$. New SiPMs from the $4^{\text {th }}$ production run were also presented. The geometric efficiency or fill factor of these new devices ranges between $44 \%$ and $76 \%$ depending on the detector geometry. Some of these new geometries are shown in figure 6. Arrays (not shown here) have also been produced. The new geometries have been developed for different applications and some effort was made to optimize the fill factor. Tests of these new devices is under way and preliminary results show breakdown voltages, gains and dark count rates similar to the baseline SiPMs. 

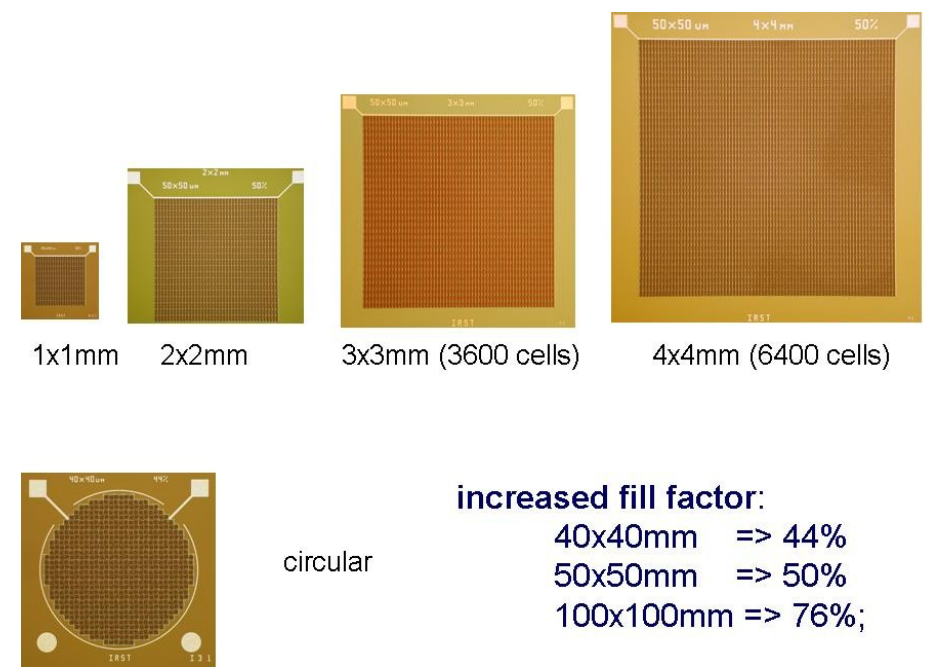

$$
\begin{aligned}
& \text { increased fill factor: } \\
& \begin{array}{l}
40 \times 40 \mathrm{~mm}=>44 \% \\
50 \times 50 \mathrm{~mm}=>50 \% \\
100 \times 100 \mathrm{~mm}=>76 \%
\end{array}
\end{aligned}
$$

Figure 6: Some new SiPM models produced by IRST

\section{Applications}

Given our initial objective of optimizing the application SiPMs for detectors of interest to particle physics, application of the devices to such detectors has been tested soon after they became available. Such tests furnish essential feedback for application - specific development.

The first application test undertaken by our group was for the read-out of the muon detector scintillator strips at the Fermi National Accelerator Laboratory test beam experiment T956 . The purpose of this experiment is to test the performance of planes of $4 \mathrm{~cm} \times 1 \mathrm{~cm} \times(1-2) \mathrm{m}$ polystyrene scintillator strips for a muon detection. The light generated in the scintillator strips by passing muons is collected by a single wavelengthshifter (wls) fiber running down the center of the scintillator strip, which is read out at on or both ends by one pixel of a multianode photmultiplier (MAPMT). For our test, IRST SiPMs were used instead of MAPMTs (see figure 7) and data was take with $120 \mathrm{GeV}$ protons.
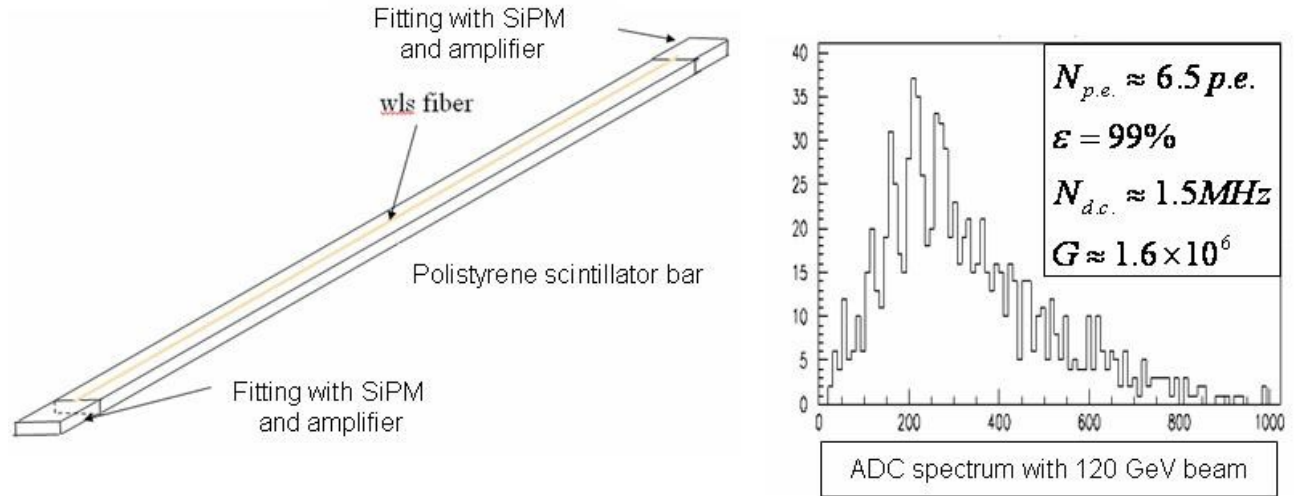
Figure 7:Scintillator bar read out on both ends by IRST SiPMs (left) and an ADC spectrum from data taken with $120 \mathrm{GeV}$ proton beam

Despite the low geometric efficiency of the baseline-model SiPMs used, full detection efficiency was achieved and the mean number of photo-electrons collected were comparable to those seen by the MAPMTs. Dark count backgrounds around $\sim 15 \%$ were compatible with the 160 ns ADC integration gate and the $\sim 1 \mathrm{MHz}$ dark count. Future work is being planned with a counter plane fully equipped with the new (circular area) SiPMs shown in figure 6.

The use of IRST SiPMs has also been tested at Frascati for the read-out of scintillator tiles with embedded wls fiber for light collection and extensive tests are being planned to investigate the use of IRST SiPMs for scintillator - based calorimetry.

\section{Conclusion}

A recently instituted collaborative effort to develop IRST SiPMs for application to detectors for particle- and astro-physics is under way and the first results of this collaboration have been reported . Static and dynamic measurements of various IRST devices compare well with analogous measurements at IRST and the results so far obtained reveal progress in the reduction of the dark count rate. We look forward to the continuation and extension of our program of characterization.

IRST has also made considerable progress in the application - specific design and on their geometric efficiency of SiPMs and plans for their application to calorimetry and muon detectors are under way.

\section{Acknowledgements:}

We are grateful to IRST and to the technical staff of the INFN (sezione di Trieste) and the University of Udine and to Fermilab for their assistance in the execution of the work reported. This work was supported by the INFN.

\section{References}

[1] P.P. Antich et al. Nucl. Instr. and Meth. A 389 (1997) 491.

[2] V. Saveliev, V. Golovin, Nucl. Instr. and Meth. A 442 (2000) 223.

[3] C. Piemonte, Nucl. Instr. and Meth. A 568 (2006) 224.

[4] N.Dinu et al., Nucl. Instr. And Meth. A 572 (2007) 422. 
[5] C. Piemonte et al., IEEE Trans. Nuclear Science, vol. 54, No. 1 (2007) 1.

[6] SiPMs distributed by Photonique SPA and Forimtech SPA, both inGeneva, Switzerland and both MRS devices.

[7] Agilent ABA-52563 GHz RFIC Amplifier.

[8] V. Bonvicini, First workshop on photon detection 13 - 14 June 2007, Perugia (to be published in a special edition of Nuovo Cimento)

[9] C. Piemonte V. Bonvicini , First workshop on photon detection 13 - 14 June 2007, Perugia (to be published in a special edition of Nuovo Cimento) 